\title{
Protein Quantification at the Single Vesicle Level Reveals That a Subset of Synaptic Vesicle Proteins Are Trafficked with High Precision
}

\author{
Sarah A. Mutch, ${ }^{1}$ Patricia Kensel-Hammes, ${ }^{2}$ Jennifer C. Gadd, ${ }^{1}$ Bryant S. Fujimoto, ${ }^{1}$ Richard W. Allen, ${ }^{1}$ Perry G. Schiro, ${ }^{1}$ \\ Robert M. Lorenz, ${ }^{1}$ Christopher L. Kuyper, ${ }^{1}$ Jason S. Kuo, ${ }^{1}$ Sandra M. Bajjalieh, ${ }^{2}$ and Daniel T. Chiu ${ }^{1}$ \\ Departments of ${ }^{1}$ Chemistry and ${ }^{2}$ Pharmacology, University of Washington, Seattle, Washington 98195
}

\begin{abstract}
Protein sorting represents a potential point of regulation in neurotransmission because it dictates the protein composition of synaptic vesicles, the organelle that mediates transmitter release. Although the average number of most vesicle proteins has been estimated using bulk biochemical approaches (Takamori et al., 2006), no information exists on the intervesicle variability of protein number, and thus on the precision with which proteins are sorted to vesicles. To address this, we adapted a single molecule quantification approach (Mutch et al., 2007) and used it to quantify both the average number and variance of seven integral membrane proteins in brain synaptic vesicles. We report that four vesicle proteins, SV2, the proton ATPase, Vglut1, and synaptotagmin 1, showed little intervesicle variation in number, indicating they are sorted to vesicles with high precision. In contrast, the apparent number of VAMP2/synaptobrevin 2, synaptophysin, and synaptogyrin demonstrated significant intervesicle variability. These findings place constraints on models of protein function at the synapse and raise the possibility that changes in vesicle protein expression affect vesicle composition and functioning.
\end{abstract}

\section{Introduction}

Neurotransmitters are released at synapses via the fusion of transmitter-containing (synaptic) vesicles with the presynaptic plasma membrane. Formed during endocytosis, synaptic vesicles contain all the machinery they need to fill with neurotransmitter, associate with synaptic sites at the plasma membrane, and fuse in response to elevated calcium. Most of the protein constituents of synaptic vesicles have been identified and their contribution to vesicle functioning has been analyzed by genetic approaches. The copy number of the majority of vesicle proteins was recently estimated using biochemical approaches (Morciano et al., 2005; Burré et al., 2006; Takamori et al., 2006).

What is not known is the extent to which vesicles vary in protein composition and thus the consistency with which proteins are sorted to vesicles. Studies tracking the location of fluorescent vesicle proteins suggest that they freely exchange with excess protein on the plasma membrane (Fernández-Alfonso et al., 2006; Wienisch and Klingauf, 2006). This exchange is consistent with the dynamic modulation of vesicle protein number in response to changing synaptic conditions. Indeed, functional differences consistent with variance in protein number have been

Received July 22, 2010; revised Nov. 12, 2010; accepted Nov. 23, 2010.

This work was supported by a grant from the National Institute of Neurological Disorders and Stroke (NS052637 to D.C. and S.B.). The monoclonal antibody directed against Vglut 1 that was developed by and/or obtained from the University of California, Davis (UC Davis)/National Institutes of Health (NIH) NeuroMab Facility, was supported by NIH GrantU24NS050606 and maintained by the Department of Neurobiology, Physiology and Behavior, College of Biological Sciences, UC Davis, California. We thank Prof. Reinhard Jahn for his input on this project and Prof. Rachel Wong for comments on the manuscript.

Correspondence should be addressed to either of the following: Sandra M. Bajjalieh at the above address, E-mail: bajjalie@u.washington.edu; or Daniel T. Chiu at the above address, E-mail: chiu@chem.washington.edu. DOI:10.1523/JNEUROSCI.3805-10.2011

Copyright $\odot 2011$ the authors $\quad 0270-6474 / 11 / 311461-10 \$ 15.00 / 0$ reported (Gracz et al., 1988), including differences in synaptic vesicle fusion within single synapses (Trommershäuser et al., 2003; Müller et al., 2010). Likewise, differences in endocytotic machinery suggest that protein content may vary between vesicles (Hayashi et al., 2008). In contrast, other studies support the conclusion that vesicles are either recycled intact (Ceccarelli et al., 1973; Murthy and Stevens, 1998; Alés et al., 1999; Klyachko and Jackson, 2002; Aravanis et al., 2003; Richards et al., 2005; Wu et al., 2005; He et al., 2006) (for review, see $\mathrm{He}$ and $\mathrm{Wu}, 2007$ ), or that subsets of proteins remain clustered after exocytosis (Willig et al., 2006). This suggests little change in vesicle protein composition and is consistent with protein interactions producing a precise protein stoichiometry in vesicles.

To address this question we developed an approach to quantify proteins in isolated, single synaptic vesicles and used it to quantify seven major membrane proteins of rat brain synaptic vesicles. The approach combines organelle purification with immunolabeling, microfluidics, and total-internal-reflectionfluorescence (TIRF) microscopy. Using immunofluorescence to quantify microscopic organelles requires complete and verifiable labeling, reproducible image collection, an analysis method that can resolve aggregates from isolated structures, a calibration approach that accounts for the variation in the system, and a statistical method to fit fluorescent intensity distributions obtained with labeled organelles to calibration distributions. The method we describe addresses each of these issues. Our results suggest that vesicle proteins vary in the precision with which they are sorted to or organized within synaptic vesicles and thus in their potential to contribute to either synaptic plasticity or pathological processes. 


\section{Materials and Methods}

Antibodies. Agarose beads conjugated with goat anti-mouse (GAM), goat anti-rabbit (GAR), mouse IgG, or rabbit IgG were obtained from SigmaAldrich. Anti-synaptic vesicle protein 2 monoclonal antibody (SV2 mAb) (Buckley and Kelly, 1985) and anti-synaptotagmin polyclonal antibody (SYT pAb) (Schivell et al., 1996) recognize epitopes in the cytoplasmic domains of these proteins. Anti-synaptophysin $\mathrm{mAb}$ (SYP mAb) and anti-VAMP2/synaptobrevin $2 \mathrm{mAb}$ (VAMP2 mAb) were obtained from Lifespan Biosciences. The polyclonal antibody directed against the vesicular proton pump and SYG $\mathrm{mAb}$ (anti-synaptogyrin $\mathrm{mAb}$ ) was obtained from Synaptic Systems. The monoclonal antibody directed against vesicular glutamate transporter 1 (Vglut1) was developed by and/or obtained from the University of California, Davis/National Institutes of Health NeuroMab Facility. SYT1 mAb was obtained from Millipore Bioscience Research Reagents/Millipore. Fluorescently labeled secondary antibodies (GAM Alexa-488, GAM Alexa-635, GAR Alexa-488, and GAR Alexa-635) were from Invitrogen/Invitrogen. GAM horseradish peroxidase conjugate and GAR horseradish peroxidase conjugate were from Zymed Laboratories. Supplemental Table S1, available at www.jneurosci. org as supplemental material, lists the combinations and concentrations of antibodies used. Supplemental Figure S1, available at www.jneurosci. org as supplemental material, shows Western blot analyses for each antibody used. Each antibody had a band associated with the appropriate molecular weight for the protein being probed.

Isolation of vesicles from brain homogenates. Ten frozen Sprague Dawley rats, 7-8 weeks old, mixed gender brains (Pellfreeze) were pulverized into a fine powder by blending in liquid nitrogen. The powder was resuspended in $85 \mathrm{ml}$ of homogenization buffer $(0.3 \mathrm{~m}$ sucrose, $50 \mathrm{~mm}$ HEPES, pH 7.4, 2 mm EGTA) and homogenized using a Teflon-glass homogenizer. The homogenate was centrifuged at $100,000 \times g$ for 1 hat $4^{\circ} \mathrm{C}$. The resulting supernatant was loaded onto a $10 \mathrm{ml} 0.6 \mathrm{M} / 1.5 \mathrm{M}$ sucrose step gradient, then centrifuged at $260,000 \times g$ for 2 hat $4^{\circ} \mathrm{C}$. Synaptic vesicles were collected from the interface of the $0.6 \mathrm{M} / 1.5 \mathrm{M}$ sucrose steps. Isolated synaptic vesicles were diluted to $\sim 3 \mathrm{mg} / \mathrm{ml}$ as determined using Bio-Rad protein assay kit with bovine serum albumin as a standard. Vesicles were frozen and stored at $-80^{\circ} \mathrm{C}$ for up to 6 months.

Preparation of vesicles for fluorescence imaging. Synaptic vesicles were exchanged into PBS by gel filtration using a $3 \mathrm{ml}$ BioGel P10 (Bio-Rad) column. Fifty micrograms of vesicle protein was incubated for $5 \mathrm{~h}$ at $4^{\circ} \mathrm{C}$ with the antibody used for quantification (usually a monoclonal antibody). We note that an increase in labeling was not obtained with longer labeling times (data not shown). The sample was then incubated with anti-IgG-agarose (Sigma) to remove excess primary antibody. The supernatant containing synaptic vesicles was transferred to a new tube and incubated overnight at $4^{\circ} \mathrm{C}$ with Alexa-488-conjugated secondary antibody (Invitrogen). We determined that an overnight (12 h) incubation was necessary to obtain a saturated signal (data not shown). Vesicles were then incubated with a second confirmatory antibody (all vesicles colabeled with SV2 polyclonal Ab, except for SV2 primary labeled vesicles that were labeled with synaptotagmin polyclonal Ab, and the Vesicular Proton Pump primary labeled vesicles that were colabeled with SV2 monoclonal $\mathrm{Ab}$ ) and the corresponding Alexa-635-conjugated secondary antibody. We allowed a minimum of $1 \mathrm{~h}$ at $4^{\circ} \mathrm{C}$ for each step. Synaptic vesicles were then incubated with anti-goat-IgG-agarose (Sigma) to remove excess secondary antibody from the solution. The specificity of each antibody used was tested by Western blot. Every analysis included both fully labeled and singly labeled vesicles.

Fabrication of micro-channels. To generate imaging "chips," four 1-mm-wide and 300- $\mu \mathrm{m}$-high straight channels were fabricated in polydimethylsiloxane (PDMS) with rapid prototyping (Fiorini and Chiu, 2005) and sealed irreversibly to a borosilicate glass coverslips (supplemental Fig. S2a, available at www.jneurosci.org as supplemental material). Briefly, a high-resolution mask was generated from a computer-aided drawing file imprinted with the channel design. The mask was used in contact photolithography with SU-8 photoresist (MicroChem) to create a master that consisted of the positive features of the 1-mm-wide and $300-\mu \mathrm{m}$-high straight channel on silicon wafer. From the master, a PDMS chip containing channels was molded. To form the reservoirs at both ends of the microchannel, a punch made from aluminum tubing ( $~ 5 \mathrm{~mm}$ diameter) was used to "drill" holes in the PDMS (supplemental Fig. S2a, available at www.jneurosci.org as supplemental material). The PDMS chip was sealed irreversibly to a borosilicate glass coverslip by oxidizing the PDMS surface in oxygen plasma. Before sealing, the glass coverslip was cleaned thoroughly by boiling it for $1.5 \mathrm{~h}$ in a 3:2:1 mixture of water, ammonium hydroxide, and $30 \%$ hydrogen peroxide, followed by thorough rinsing with ultrapure water. Channel-to-channel and chipto-chip variability was found to be $\sim 5 \%$ (supplemental Fig. S2, available at www.jneurosci.org as supplemental material).

Optical setup. Total internal reflection fluorescence was generated by directing the output of the 488 and $633 \mathrm{~nm}$ lasers off axis, via a polychroic mirror, into the outer edge of the back aperture of a high numerical aperture (NA) objective (NA 1.45), thereby creating total internal reflection at the interface of the coverslip and solution (Fig. 1a). Molecules or vesicles immobilized on the surface of the coverslip were excited by the $\sim 300$-nm-thick evanescent field generated by total internal reflection. Fluorescence emission was collected by the objective then split by a dichroic filter into two-color channels, with each channel filtered by its respective bandpass filter and imaged onto a high sensitivity charge-coupled device (CCD) camera. A dual-view was placed in the imaging path to allow for simultaneous two-color imaging. The dualview splits the signal from the red and green fluorescence onto half of the camera CCD.

Fluorescent imaging of single vesicles and single antibodies. Vesicles were introduced by gravity-driven flow into micro-channels bonded to glass coverslips. Gravity-driven flow was induced by placing $100 \mu \mathrm{l}$ of ultrapure water into the inlet reservoir. Water was then replaced with imaging buffer (PBS for synaptic vesicles). To remove non-adsorbed vesicles, 200 $\mu \mathrm{l}$ of PBS was subsequently flowed through the channel. The coverslips were imaged with a home-built TIRF microscope. Samples were excited simultaneously with a $488 \mathrm{~nm}$ light from a $20 \mathrm{~mW}$ solid-state laser (Coherent) and with a $633 \mathrm{~nm}$ light from a $10 \mathrm{~mW}$ HeNe laser (Coherent). The power of the 488 and $633 \mathrm{~nm}$ light after passing through the objective was $88 \mu \mathrm{W}$ and $118 \mu \mathrm{W}$, respectively. Exposure time was $300 \mathrm{~ms}$. Images were split with a dual-view (Photometrics) and acquired with a PhotonMax EMCCD camera (Princeton Instruments). Single-antibody images were obtained using a procedure similar to the one for imaging individual synaptic vesicles. A $0.01 \mathrm{mg} / \mathrm{ml}$ mixture of primary-secondary antibody complexes was diluted 1:1000 in high quality (MilliQ, ultrapure) water and flowed through the channel. Antibody complexes were allowed to adsorb onto the floor of the channel for $\sim 1 \mathrm{~min}$.

Fluorescence intensity measures are subject to variation in laser power and to photobleaching. We assessed the stability of the laser output over time and found it to be stable after $30 \mathrm{~min}$ (supplemental Fig. S3, available at www.jneurosci.org as supplemental material). To determine the extent of photobleaching during data acquisition, we measured the intensity of fluorescently labeled vesicles over $1500 \mathrm{~ms}$ as shown in supplemental Figure S4, available at www.jneurosci.org as supplemental material; we did not see significant photobleaching. Note that we used a $300 \mathrm{~ms}$ exposure in all of our studies. Thus, samples did not undergo significant photobleaching within the time course of the data collection.

Fluorescent puncta analysis. We developed an image-processing program in Matlab to analyze fluorescent images. To determine the total intensity of spots (puncta), the program fit the intensity of pixels in each puncta (less background intensity) to a two-dimensional Gaussian distribution. The width of the fluorescent puncta (defined as the point where the intensity drops to $1 / e^{2}$ of its peak intensity) was used to identify spots that exceeded the measured resolution limit of our imaging setup $(\sim 2.3$ pixels). These were rejected as likely aggregates. The total intensity of accepted spots with a signal-to-noise ratio $>3$ was obtained by integrating the total intensity of the puncta. In addition we required that vesicle spots exhibit two-color overlay (to ensure that we have two colocalized vesicle proteins).

Data analysis and fitting. The average number of proteins per vesicle was measured by dividing the average intensity value of fully labeled vesicles by the average intensity value of singly labeled vesicles. The range in number of proteins per vesicle was determined by fitting the fully labeled vesicle data to scaled single-antibody distributions (calibration 


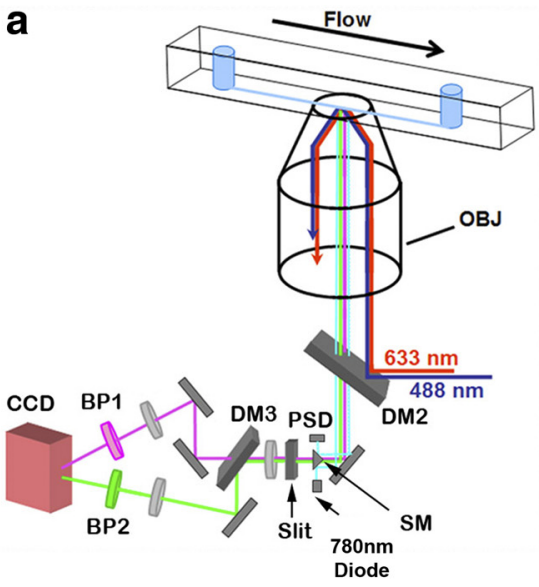

b

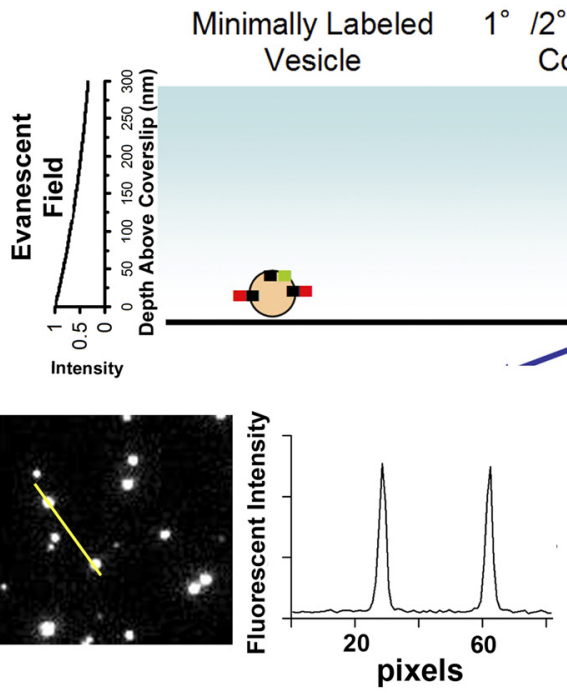

d

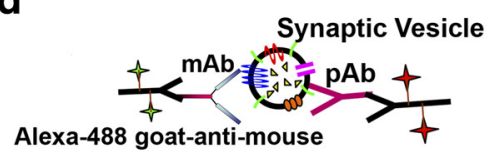

Alexa-635 goat-anti-rabbit

e

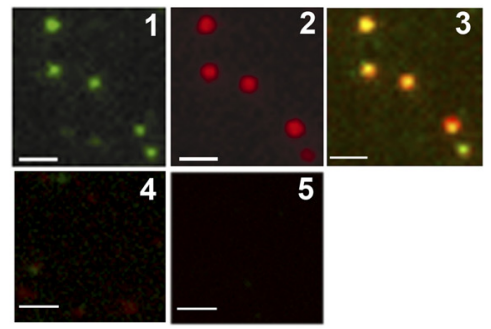

Figure 1. Experimental configuration for imaging single molecules and synaptic vesicles using TIRF microscopy. $\boldsymbol{a}$, Schematic of the system in which samples were imaged in microfluidic channels. Fluorescent samples were flowed into the channels and excited by green $(488 \mathrm{~nm})$ and red $(633 \mathrm{~nm})$ lasers simultaneously. Images were focused using a CRIFF (continuous reflective-interface feedback focus system; depicted as a light blue line). Emitted light was split with a dual-view imaging module and collected by a cooled, high-sensitivity CCD camera. OBJ, Objective; DM, dichoric mirror; SM, scanning mirror; PSD, position-sensitive diode; L, lens; BP, bandpass filter; CCD, camera. $\boldsymbol{b}$, Diagram of the TIRF-imaging environment. Minimally labeled vesicles (left), single antibody complexes (middle), and fully labeled vesicles (right). The decay of laser intensity is illustrated in the plot to the left of the drawing, which we measured to be $\sim 300 \mathrm{~nm}$ (1/e). c, High signal-to-noise ratio obtained with antibody-labeled synaptic vesicles. Labeled vesicles were adsorbed onto the floor of a microchannel and imaged (left). The linescan plots the intensity of each pixel along the indicated line.d, Schematic of the two-color labeling scheme in which synaptic vesicles were labeled with antibodies directed against two different synaptic vesicle proteins, each of which was detected with a different colored fluorescent secondary antibody. $\boldsymbol{e}$, Verification of vesicle labeling. 1, 2, Sample image of synaptic vesicles labeled with anti-SV2 and goat-anti-mouse Alexa-488 (green, 1) and anti-synaptotagmin 1 and goat-anti-rabbit Alexa-635 (red, 2). 3, Two-color overlay in which colocalization of the two probes appears yellow.4, Sample in which primary antibodies were excluded from the labeling protocol. 5, Sample in which vesicles were excluded from the labeling protocol. Scale bars, $1 \mu \mathrm{m}$.

icle data were fit with $10-30$ of the calibration distributions and the best fit determined using the simulated annealing minimization program AMEBSA (Press, 2001). It is important to note that although the mean of the two distributions (the singly labeled vesicles and single antibody distribution) is different, the shape of the two distributions stayed constant, thus the single-antibody data can be scaled and used to fit the vesicle distribution.

Immunoblotting. Two micrograms of vesicle protein was diluted 1:1 with SDS-PAGE sample loading buffer. The samples were loaded onto a $4-15 \%$ Tris- $\mathrm{HCl}$ gradient gel (Bio- $\mathrm{Rad}$ ) and resolved for $45 \mathrm{~min}$ at $200 \mathrm{~V}$. Proteins were transferred to PVDF (Millipore) and probed with antibodies to synaptic vesicle proteins. Antibody binding was detected with horseradish peroxidase-conjugated secondary antibodies that were detected with DuraWest substrate (Pierce Biotechnology). Chemiluminescence was quantified using a Kodak 440 imaging station.

Atomic force microscopy imaging of synaptic vesicles. Fluorescent antibody-labeled vesicles were flowed over mica, allowing them to come in contact and nonspecifically adsorb onto the surface. Images were acquired while the vesicles were immersed in PBS using tappingmode atomic force microscopy (AFM), and the diameters of the vesicles were measured using contact-mode AFM. The diameters were calculated using the measured circumference from each synaptic vesicle.

\section{Results Imaging proteins on single synaptic vesicles}

We quantified seven major membrane proteins in purified rat brain synaptic vesicles by labeling them with fluorescent antibodies and imaging them using TIRF microscopy (Fig. 1). TIRF microscopy was chosen because it reduced background fluorescence and produced a signal-to-noise ratio that was higher than that obtained with standard epifluorescence. The vesicle purification and labeling process is outlined in supplemental Figures S5 and S6, available at www. jneurosci.org as supplemental material. To unambiguously detect synaptic vesicles in the synaptic vesicle fraction, we used a two-antibody, two-color colocalization approach (Fig. 1d). Antibodies used to quantify a protein of interest (target antibodies) were monoclonal to ensure a 1:1 antibody to protein binding ratio (with the exception of a polyclonal directed against a peptide fragment of the Voa subunit of the $\mathrm{H}^{+}$/ATPase). Target antibody binding was detected with sec-

distributions) multiplied by integer values (Mutch et al., 2007). We found that scaling was necessary because although the shape of the intensity distributions obtained with isolated antibodies were stable and the same as those observed for singly labeled vesicles, the intensities for the isolated antibodies were different in average value. Fully labeled ves- ondary antibodies labeled with Alexa Fluor 488 (green). Using antibody binding as a quantitative measure relies on saturated labeling of the proteins being quantified and on a reproducible antibody-to-protein ratio. The concentration of the target anti- 
body needed to saturate (fully label) a vesicle was determined for each target antibody by measuring the average intensity of vesicles labeled with a range of target antibody concentrations. Vesicles were considered fully labeled when the average intensity per fluorescent spot (i.e., vesicle) reached a stable high plateau. Figure 2 shows antibody dilution curves for the target antibodies used in these studies. The green arrow indicates the antibody concentrations used to produce fully labeled vesicles. Antibodies directed against a second vesicle protein (usually a polyclonal) were used for synaptic vesicle verification and were detected with secondary antibodies labeled with Alexa Fluor 635 (red).

Labeled synaptic vesicles were introduced into microchannels as described in Materials and Methods, and illuminated simultaneously with two lasers (emitting light of 488 and $633 \mathrm{~nm}$ ) that generated separate images in each color. Fortuitously for our studies, we found that when labeled vesicles were introduced into channels in PBS they preferentially adhered to the glass coverslip whereas free antibody did not (supplemental Fig. S7, available at www.jneurosci.org as supplemental material), thus providing an additional purification of the preparation. Image analysis involved overlaying the two color images (Fig. 1d) and quantifying spots that exhibited two-color overlap. Control studies revealed that vesicles incubated with only secondary antibodies (no primary antibodies) did not demonstrate two-color overlay (Fig. 1e4). Likewise, samples lacking vesicles had no two-color spots and very few fluorescent objects (Fig. 1e5), whereas samples containing vesicles had many two-color spots.

We tested the integrity of vesicle labeling in a series of control studies. To assess the effects of steric hindrance affecting antibody binding, we calculated the surface area of a $40 \mathrm{~nm}$ diameter vesicle and determined that it can accommodate over 100 antibody complexes, each of which is estimated to have a diameter of $7.5 \mathrm{~nm}$. This suggests that the labeling of equally spaced vesicle proteins present at $<100$ copies/vesicle should not be affected by steric hindrance between antibody complexes. To determine the stability of antibody labeling, we measured the fluorescence of a single sample over the course of $3 \mathrm{~d}$. We found that the measured fluorescence intensity did not vary (supplemental Fig. S8, available at www.jneurosci.org as supplemental material), indicating that label degradation and dissociation were also not issues. We also checked whether measurements were antibody-dependent by comparing the results obtained with two antibodies directed against different epitopes on VAMP2 (synaptobrevin 2). Both produced similar average numbers of proteins per vesicle (supplemental Fig. S9, available at www.jneurosci.org as supplemental material; Table 1).
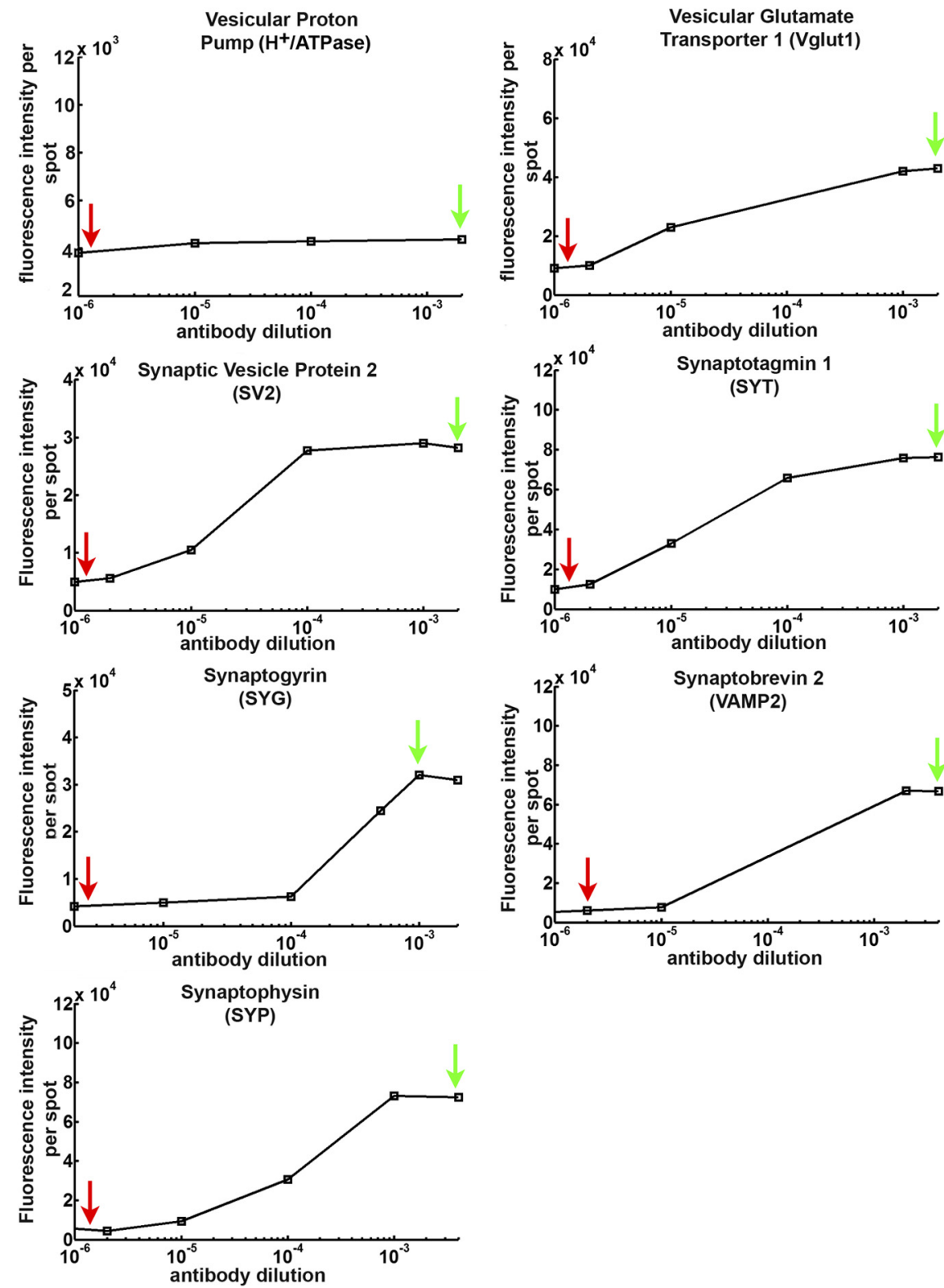

Figure 2. Determining the concentration of antibody required to produce fully and minimally labeled vesicles. Vesicles were labeled with the indicated antibody dilutions (from a stock concentration of $\sim 2 \mathrm{mg} / \mathrm{ml}$ ). Vesicles were judged to be fully labeled at antibody concentrations that produced no further increase in measured intensity per fluorescent spot (i.e., vesicle, green arrows), and to be minimally labeled at antibody concentrations that produced no further decrease in measured intensity per fluorescent spot (i.e., vesicle, red arrows).

Because synaptic vesicles are smaller than the diffraction limit of a light microscope, a single vesicle and a vesicle aggregate will produce similar images. To determine whether our samples contained a significant proportion of vesicle aggregates, we assessed our samples with multiple approaches. First, we profiled the dimensions of labeled synaptic vesicles using contact-mode AFM (Fig. 3a). This measure revealed a distribution of vesicle diameters centered at $50 \mathrm{~nm}$ (Fig. $3 b$ ). This is slightly larger than the 37-44 nm size obtained with electron-microscopy measurements (Bekkers et al., 1990), perhaps because of the tip pushing down on the vesicles causing a slight flattening while performing contact-mode AFM (Parpura et al., 1995). But it indicates that antibody labeling did not lead to vesicle aggregation. Second, we filtered labeled vesicles though a $0.11 \mu \mathrm{m}$ filter and measured the average spot intensity before and after filtration. We found that 
Table 1. Average number of seven integral membrane proteins in synaptic vesicles

\begin{tabular}{|c|c|c|c|c|c|c|c|c|}
\hline Protein & $\begin{array}{l}\text { Average number } \\
\text { per vesicle }\end{array}$ & SEM & $\begin{array}{l}\text { Samples } \\
\text { analyzed }\end{array}$ & $\begin{array}{l}\text { Total number of } \\
\text { vesicles analyzed }\end{array}$ & $\begin{array}{l}\text { Average number of } \\
\text { vesicles per sample }\end{array}$ & $\begin{array}{l}\text { Distribution } \\
\text { width }\end{array}$ & $\begin{array}{l}\text { SE of distribution } \\
\text { width }\end{array}$ & $\begin{array}{l}\text { Coefficient of variation } \\
\text { for distribution }\end{array}$ \\
\hline Proton ATPase & 1.27 & 0.19 & 6 & 8826 & 1765 & 0.49 & 0.17 & 0.38 \\
\hline SV2 & 5.04 & 0.09 & 6 & 15,906 & 2651 & 0.23 & 0.21 & 0.057 \\
\hline VGlut1 & 4.09 & 0.12 & 5 & 20,989 & 4197 & 0.29 & 0.18 & 0.057 \\
\hline Synaptotagmin 1 & 7.16 & 0.07 & 5 & 9757 & 1951 & 0.86 & 0.08 & 0.12 \\
\hline Synaptogyrin 1 & 6.80 & 0.36 & 6 & 9026 & 1504 & 1.55 & 0.71 & 0.23 \\
\hline VAMP2 & 10.53 & 0.37 & 6 & 18,954 & 3159 & 5.13 & 0.84 & 0.49 \\
\hline Synaptophysin & 13.06 & 0.83 & 6 & 10,462 & 1743 & 3.60 & 2.17 & 0.28 \\
\hline
\end{tabular}

The average was determined by dividing the average total intensity of fully labeled vesicles by the average total intensity of minimally labeled vesicles for each sample. When the average was calculated using the fitting, the same values were obtained for all proteins except Synaptophysin for which the average was 14.0. The distribution width is the SD of each distribution averaged over all samples for each protein. The SE of the distribution width is the SEM for five separate measurements for VGlut1 and Synaptotagmin and six separate measurements for the remainder of the proteins. The coefficient of variation is the distribution width divided by the average protein number per vesicle.

a
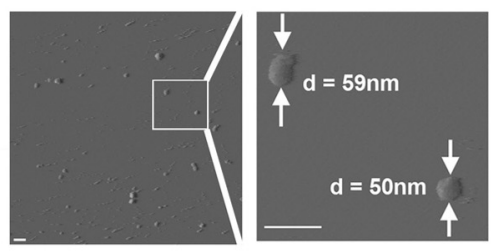

C

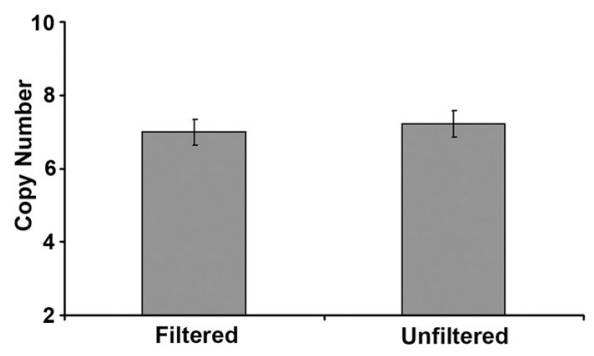

b

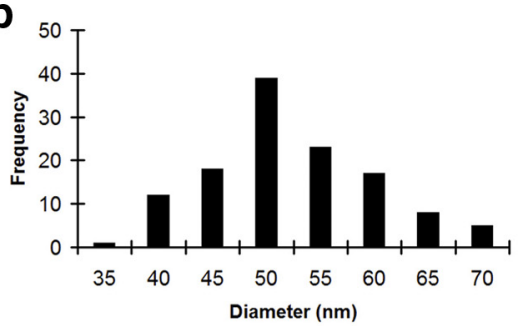

d

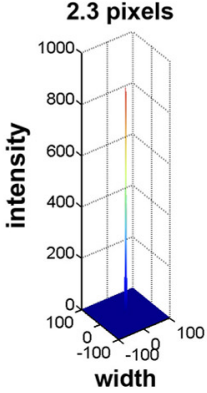

10 pixels

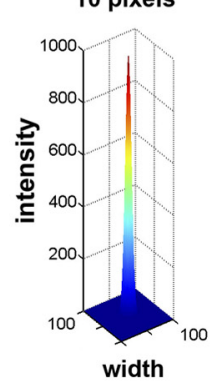

Figure 3. Verification of single vesicle samples. AFM imaging of labeled synaptic vesicles and illustrative example of microscope resolution. $\boldsymbol{a}$, AFM imaging of labeled synaptic vesicles. Shown are AFM images of antibody-labeled vesicles in solution on a mica surface. Scale bars, $100 \mathrm{~nm}$. $\boldsymbol{b}$, Histogram showing the diameter of the labeled vesicles as measured with contact-mode AFM. $\boldsymbol{c}$, Filtering of synaptic vesicles to test for vesicle aggregation. To ensure we were not imaging aggregates of vesicles, we filtered a vesicle sample through a $0.11 \mu \mathrm{m}$ pore-diameter polycarbonate filter and measured the mean intensity of the synaptic vesicles before and after filtration. Aggregates of two or more vesicles labeled with antibodies are unlikely to pass though the filter. The mean intensity did not change between the filtered and unfiltered samples. Error bars represent SE between data sets. $\boldsymbol{d}$, Left, Three-dimensional Gaussian plots of a spot at the resolution of our setup (2.3 pixels); right, plot of a spot much above our measured resolution (10 pixels).

the mean intensity was not altered (Fig. 4c). Third, we found that the intensity per fluorescent spot (i.e., vesicle) plateaus with increasing antibody concentration. This argues against antibody-induced aggregation, which would produce increasing fluorescence intensity with increasing antibody concentration. Finally, we analyzed only spots that were at the resolution limit of our microscope. Fluorescent spots were eliminated if they did not fit with a Gaussian distribution with a width (the $e^{-2}$ point) of $\leq 2.3$ pixel. Figure $3 d$ shows a comparison of two spots, one in which the width is at the resolution limit of our setup (2.3 pixels, measured with fluorescent, $60 \mathrm{~nm}$ beads), and the other in which the width is much larger (10 pixels). In our analyses the larger spot would be rejected from further analysis, thus ensuring that large aggregates were excluded from our analyses.

Images were processed using a program written in Matlab that recorded the intensity of spots that met four criteria: (1) were in focus, (2) exhibited two-color overlay, (3) had a peak intensity $>$ $3 \times$ background noise, and (4) had a width (determined as described earlier) no greater than 2.3 pixels, which we determined to be the resolution of the instrument.

\section{Calculating the average number of seven membrane proteins in brain synaptic vesicles}

To measure the average number of a synaptic vesicle protein, we determined the fluorescence intensity of vesicles on which a single protein had been labeled. To do this, we identified the concentration of each antibody that produced minimal labeling. Vesicles were considered minimally labeled when the average intensity of fluorescent spots (i.e., vesicles) reached a low plateau (Fig. 2, red arrows). Any further dilution of the target antibody resulted in a decrease in the number of fluorescent spots but not further reduction in fluorescence intensity per spot. This is consistent with there being only a single target antibody per fluorescent spot, and does not require the spots to be diffraction limited. Using minimally labeled vesicles insures that fluorescent antibodies in both calibration and test samples experience the same imaging environment. This is important when using TIRF microscopy because the illumination intensity decays as a function of distance from the coverslip (Fig. 1b), and thus the intensity of fluorophores can be influenced by their position in the evanescent field. Any variability in the number of fluorophores per target antibody complex is reflected in the average intensity of the minimally labeled vesicle samples and does not have to be accounted for separately to obtain the average number of proteins per vesicle.

We measured the average number of seven integral membrane proteins in rat brain synaptic vesicles: synaptophysin, synaptotagmin 1, SV2, VAMP2/synaptobrevin 2, Vglut1, synaptogyrin 1, and Voa of the proton ATPase (Jahn and Südhof, 1994). Integral membrane proteins are most amenable to this type of measurement, because, unlike extrinsic membrane proteins, the process of vesicle purification will not lead to changes in their association with vesicles. Each vesicle protein was independently measured at least three times in studies run in duplicate. In addition, VAMP2/synaptobrevin 2 was measured using two different antibodies. The average number of a vesicle protein was determined by dividing the average intensity of fully labeled vesicles by the average intensity of minimally labeled vesicles. We also determined the average protein number by fitting vesicle 

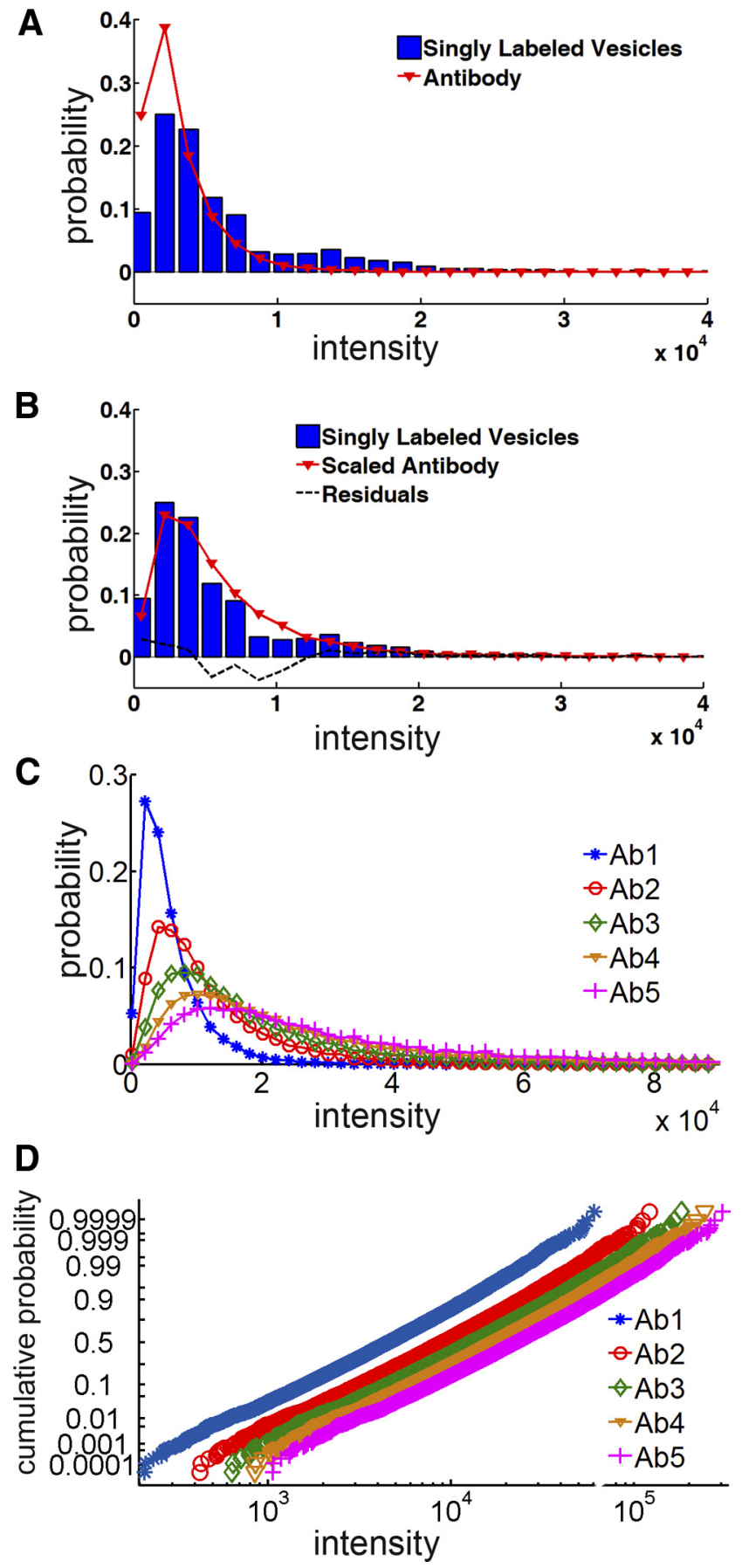

Figure 4. Procedure used to generate calibration distributions. $\boldsymbol{a}$, Intensity distributions of singleantibody complexes (red) compared with minimally labeled synaptic vesicles (blue). $\boldsymbol{b}$, Intensity distribution of single-antibody complexes from $(\boldsymbol{a})$ after it has been scaled to the same mean intensity as the minimally labeled vesicles. The goodness of fit is indicated by a reduced $\chi^{2}$ value of 1.10 . The residual of the fit (the difference between the scaled antibody and the minimally labeled vesicles) is plotted as a black dotted line. $c$, The scaled distribution of the single-antibody complexes was multiplied by integers to generate theoretical (calibration) distributions. Ab1 denotes the scaled distribution of the single-antibody complexes; Ab2 through Ab5 were obtained by multiplying the Ab1 distribution by the corresponding integers 2 through 5 . $\boldsymbol{d}$, Calibration distributions displayed as semilog cumulative probability plots. This panelillustrates that multiplication of the calibration distribution does not change the multiplicative standard deviation or shape of the distribution.

intensity distributions with their corresponding target antibody distribution. The target antibody distribution was a complex of fluorescent secondary antibody and primary antibody that was scaled to the same mean as the minimally labeled antibodies. The two approaches produced nearly identical values $( \pm 0.1)$, with the exception of synaptophysin for which the fitting program produced an average value that differed by 1 . The average number of the proteins varied from 1 to 13 (Table 1), suggesting that, on average, vesicles contain fewer than 20 copies of each of these proteins. In general the values agreed well (within a factor of 2) with those obtained by Takamori et al. (2006), who used quantitative Western analyses calibrated to purified protein standards to quantify proteins in bulk preparations of synaptic vesicles. The exception was VAMP2/synaptobrevin 2, for which we obtained an average of 10 VAMP2 proteins per vesicle. This sevenfold lower value was obtained using two different monoclonal antibodies against VAMP2, suggesting that the measure was valid.

The lower average copy number of VAMP2/synaptobrevin 2 measured in intact versus solubilized vesicles could reflect the fact that a significant proportion of VAMP2/synaptobrevin 2 exists in protein complexes (Calakos and Scheller, 1994) and thus may not be accessible to antibody labeling. To test this possibility, we measured the ratio of VAMP2/SV2 in immunoblots of solubilized, denatured vesicles. The amount of each protein was determined using recombinant protein standards run at the same time. Surprisingly, we found the results varied with the anti-VAMP2 antibody used, yielding VAMP2/SV2 ratios that ranged from 3:1 to 10:1 in the same vesicle preparations (supplemental Table 2, available at www.jneurosci.org as supplemental material). These results suggest that measures of the average number of VAMP2/ synaptobrevin 2 per vesicle may be imprecise. We note, however, that all measures indicate that VAMP2/synaptobrevin 2 is one of the more abundant vesicle proteins.

\section{Variance in vesicle protein number-how accurately are vesicle proteins sorted?}

A major advantage of imaging proteins on single vesicles is the ability to measure the intervesicle variability in protein number and thus the precision with which proteins are sorted during vesicle assembly. To do this, we adapted a method to extract the distribution of fluorophore number from the distribution of fluorescent puncta intensities (Mutch et al., 2007) (supplemental Discussion, Fitting Process, available at www.jneurosci.org as supplemental material). Optimally, we would fit distributions obtained with fully labeled vesicles to distributions obtained with minimally labeled vesicles. This, however, was not practical because calibration distributions capable of producing good fits require thousands of fluorescent puncta. This was many more than could be obtained with minimally labeled vesicles because most of the vesicles are not labeled in minimal-labeling reactions. Therefore we determined whether we could use isolated primary-secondary antibody complexes, which typically provided $>2000$ data points, to generate calibration distributions. We compared intensity distributions obtained from isolated antibody complexes [which adhered to channel surfaces under low ionic strength (supplemental Fig. S7, available at www.jneurosci. org as supplemental material)] with minimally labeled vesicles. As with other measures of single fluorescent proteins these produced lognormal distributions (Limpert et al., 2001; Kapitein et al., 2008), although they differed in average intensity (Fig. 4a). This difference likely arises from differences in the immediate environment of the fluorescent dyes in the two samples. For example, antibody complexes are on the cover glass surface, while antibodies attached to a vesicle are near other proteins and can vary in their distance from the cover glass by the diameter of the vesicle.

Scaling the antibody complex distribution by setting its average to that of the minimally labeled vesicles produced a distribu- 


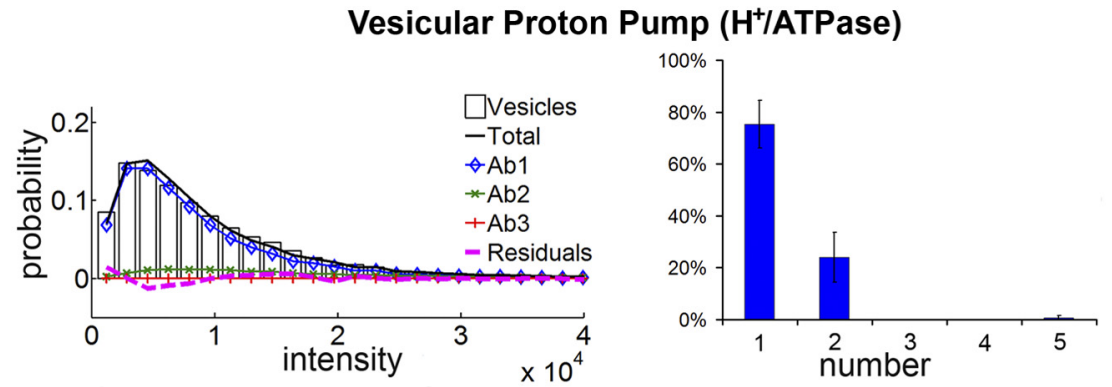

Vesicular Glutamate Transporter 1 (Vglut1)
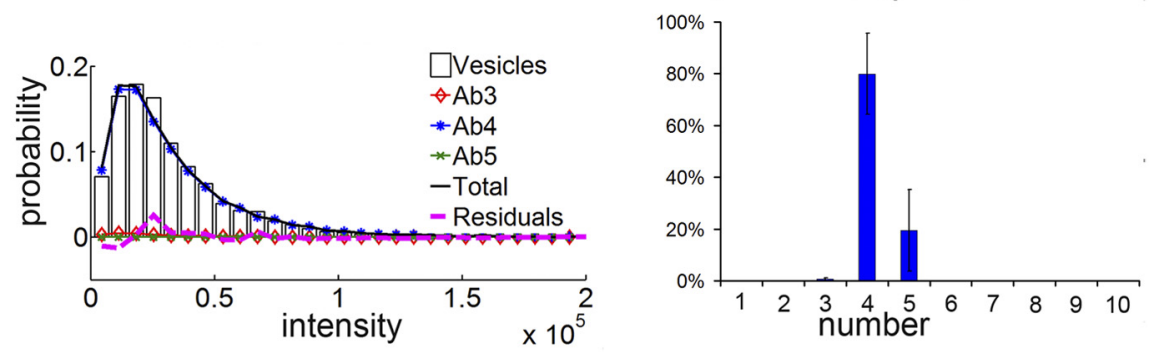

Synaptic Vesicle Protein 2 (SV2)
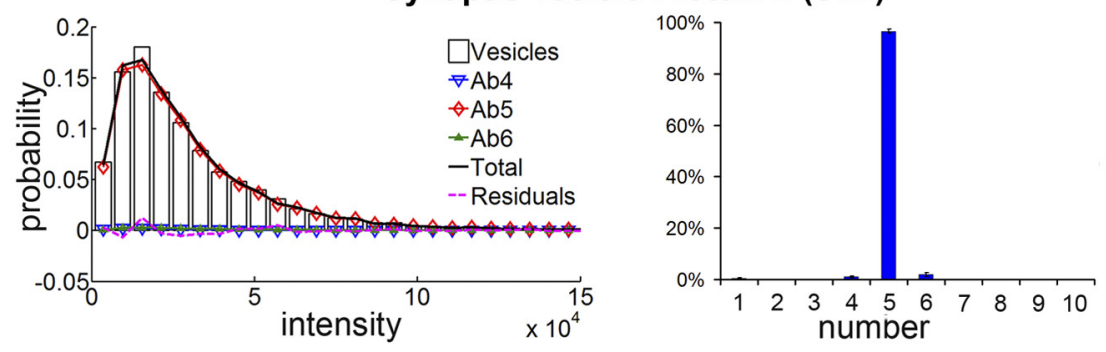

Synaptotagmin 1
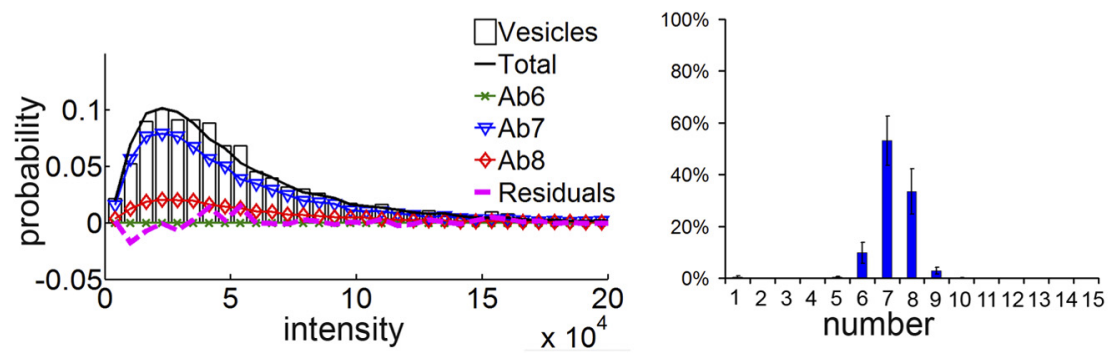

Figure 5. Quantification of monodispersed synaptic vesicle proteins. Plots of representative fits for each protein is shown on the left, and histograms showing the percentage of vesicles containing $n$ number of the indicated vesicle protein averaged across $\sim 10,000-20,000$ vesicles (see Table 1) is shown on the right. Error bars, SE between different datasets.

tion that approximated that of the minimally labeled vesicles (residual values are depicted as a dotted line in Fig. $4 b$ ). We therefore generated calibration distributions representing one target antibody complex per vesicle $(n=1)$ from antibody complex distributions scaled to the mean intensity of minimally labeled vesicles. [Additional discussion is in the text accompanying equation S4 in the supplemental material, available at www. jneurosci.org as supplemental material, and in the study by Mutch et al. (2007).] Both calibration distributions were always prepared and run at the same time as the fully labeled vesicles. To generate distributions for $n>1$, we multiplied each datum in the scaled antibody distribution by integers (Fig. 4c). Multiplying a lognormal calibration distribution to derive distributions corresponding to $n>1$ is appropriate if the distributions they represent are the same shape (Mutch et al., 2007). Figure $4 d$ shows the scaled antibody distributions as a lognormal cumulative distribution plot. This figure illustrates that the distribution shape does not change when multiplied (Mutch et al., 2007).

Factors that could influence the observed shape of calibration distributions and thus lead to spurious results include variability in the number of fluorophores per secondary antibody and variability in the number of secondary antibodies bound to each primary antibody. To address these possibilities, we compared the multiplicative standard deviation $\left(\sigma^{\star}\right)$, which reflects the shape of lognormal distributions (Limpert et al., 2001), between fluorescence intensity distributions obtained with labeled primary-secondary antibody complexes, isolated single secondary antibodies, and single fluorophores (as measured by sequential photobleaching; see supplemental Material, available at www. jneurosci.org). The resulting $\sigma^{*}$ values and their SEs were $0.63 \pm 0.05$ for the antibody complexes, $0.61 \pm 0.02$ for the isolated secondary antibodies, and $0.62 \pm 0.02$ for the single fluorophores. The similarity in $\sigma^{\star}$ indicates that neither variation in fluorophore number per antibody nor in number of secondary antibodies per complex contributes significantly to the width of the intensity distributions. While the number of fluorophores per secondary antibody is presumably not identical, the results indicate that any variation therein has a small effect on our results (see further discussion in supplemental text-Calibration Distributions, available at www. jneurosci.org). Therefore, multiplication of calibration distributions provides an adequate representation of the intensity distributions for $n>1$ proteins per vesicle.

Fluorescence intensity distributions obtained from each sample of fully labeled vesicles were independently fit to calibration distributions and fitting results were averaged. Representative fits for each protein are shown in Figures 5 and 6. Data from at least five separate runs were averaged and depicted as histograms that plot the percentage of vesicles containing $n$ number of each protein.

We found that vesicle proteins fell into two classes, those whose intensity distributions were fit by three or fewer calibration distributions and those that required more than three calibration distributions to obtain a good fit. We term proteins for which fewer than three distributions describe $>90 \%$ of the vesicles as being "monodispersed" and those for which $>3$ distributions are required to account for $90 \%$ of the vesicles as "polydispersed."

An example of a monodispersed protein is SV2. For intensity distributions obtained from synaptic vesicles labeled with antiSV2 the program was allowed 10 calibration distributions $(n=1$ through $n=10$ ) to fit the data. The vesicle data were fit best by a hybrid distribution that was $1 \% n=4,97 \% n=5$, and $2 \% n=6$. An example of a polydispersed protein is synaptogyrin. Distribu- 
tion obtained from anti-synaptogyrinlabeled vesicles required more than five calibration distributions to account for 95\% of the distribution. In this case, the vesicle data were fit best by a hybrid distribution that was $11 \pm 8 \% n=5,29 \pm$ $10 \% n=6,30 \pm 8 \% n=7,7 \pm 4 \% n=8$, and $18 \pm 11 \% n=9$.

Four of the seven proteins analyzed were relatively monodispersed (Fig. 5). This included the proton ATPase for which $n=1$ fit $75 \pm 9 \%$ of the vesicle data, Vglut1 for which $n=4$ fit $80 \pm 16 \%$, and synaptotagmin 1 for which $n=7$ and $n=$ 8 fit $\sim 90 \%$ of the vesicle data. The most monodispersed protein was SV2 for which on average $97 \pm 2 \%$ of the vesicle data were fit by $n=5$. We note that the low variability observed for these proteins is consistent with complete labeling by primary antibodies, because incomplete labeling would give rise to greater variability in apparent number. Thus it appears that a cohort of vesicle proteins varies little between vesicles.

Three of the seven vesicle proteins we analyzed were polydispersed-synaptogyrin, VAMP2/synaptobrevin 2, and synaptophysin (Fig. 6). VAMP2/synaptobrevin 2 required $>9$ calibration distributions to obtain the best fit $(8 \pm 5 \% n=6,25 \pm 11 \%$ $n=7,15 \pm 5 \% n=8,11 \pm 4 \% n=9,13 \pm$ $4 \% n=10$, and $6 \pm 2 \% n=11$ ). Synaptogyrin required $>5$ calibration distributions to obtain a good fit. Synaptophysin was the most polydispersed protein, requiring $>14$ calibration distributions to obtain a good fit.

We note that the bimodality of the combined data was not seen in individual experiments. This indicates greater variability between runs, which suggests that antigen accessibility was significantly more variable for synaptophysin than for other proteins. The absolute monodispersity or polydispersity of proteins is reflected in the SD in protein number (Table 1). When viewed in this way, it becomes apparent that synaptophysin and VAMP2/synaptobrevin 2 are the most polydispersed of the vesicle proteins we measured. We note with interest that these two proteins are reported to form a complex in vivo (Calakos and Scheller, 1994). This raises the possibility that the apparent variability in protein number measured for these proteins actually reflects changes in antibody accessibility because of variance in protein complex formation. Thus, for this subset of vesicle proteins, single vesicle quantification may measure a combination of number and conformation/interaction state.

To assess the possibility that proteins with smaller average numbers simply appear more monodisperse than proteins with larger average numbers because of the precision with which we can fit the distributions, we calculated the coefficient of variation for each of the protein distributions (Table 1). If the observed polydispersity for proteins with larger average numbers was primarily attributable to an increasing difficulty in fitting their distributions, we would expect that using the coefficient of variation would eliminate most of the differences between different proteins. This is not observed. Based on the coefficient of variation, the three most monodispersed proteins are VGlut 1, SV2, and

\section{Synaptogyrin}

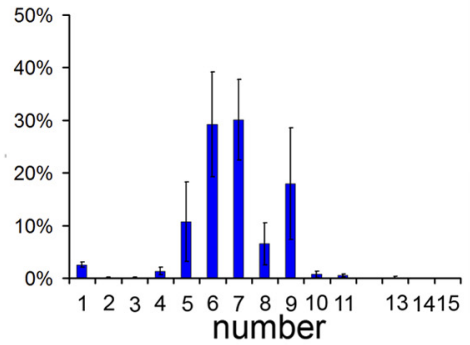

\section{Synaptobrevin 2 (VAMP2)}

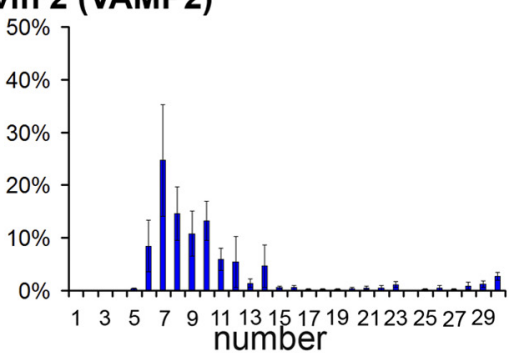

Synaptophysin

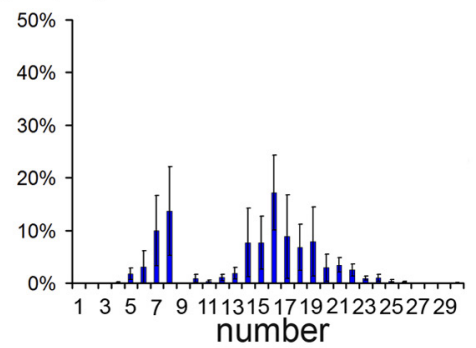
number

Figure 6. Quantification of polydispersed synaptic vesicle proteins. Plots of representative fits for each protein is shown on the left, and histograms showing the percentage of vesicles containing $n$ number of the indicated vesicle protein averaged across $\sim 10,000-20,000$ vesicles (see Table 1) is shown on the right. Error bars, SE between different datasets.

SYT. This indicates that monodispersity is not masked when proteins are present in higher numbers.

\section{Discussion}

Quantitative single vesicle imaging provides information on the variance in vesicle composition

A major advantage of quantifying native protein in isolated vesicles is that it provides information on the distribution of protein number, and with it information on the consistency of vesicle assembly. Thus the approach we describe provides a way to measure disruptions in protein sorting that may accompany neuropathological conditions. Changes in vesicle protein expression have been noted in several nervous system pathologies, including schizophrenia (Mirnics et al., 2000), epilepsy (Matveeva et al., 2003, 2007, 2008), and Alzheimer's disease, where changes in vesicle protein expression precede loss of synapses and changes in cognitive functioning (Yao et al., 2003; Yao, 2004). The technique described here will provide a means for identifying changes in the precision of protein sorting in animal models of disease and with changes in protein expression.

A potential limitation of the approach follows from its strength-the fact that proteins are measured in situ. Because proteins in their native setting are often in complexes with other proteins, measured polydispersity in protein number cannot be distinguished from variability in protein interactions, which have the potential to block antibody binding. Changes in the apparent 
polydispersity of a protein therefore indicate changes in either protein number or protein conformation/interaction state. Despite this, the technique provides a powerful tool for assessing differences in vesicle protein composition across animals of varying genotypes and in response to environmental changes.

\section{Functional implications of vesicle protein number and variability}

The number and variability of each protein in synaptic vesicles has implications for hypotheses of protein action at the synapse. Four of the seven proteins analyzed, SV2, $\mathrm{H}^{+}$/ATPase, Vglut1, and synaptotagmin, varied little in number of copies per vesicles. This was seen despite the fact that the vesicles analyzed represented all neurotransmitter classes. This finding supports a model in which protein complexes with a fixed stoichiometry interact with clathrin adaptor proteins in a stereotypical manner. In this way, vesicle protein content could be encoded into protein interactions that result in the self-assembly of vesicles with uniform protein content. This model is consistent with the report that SV2, the most monodispersed of the vesicle proteins, regulates the amount of synaptotagmin in synaptic vesicles (Yao et al., 2010).

Our finding that the glutamate transporter Vglut1 is relatively monodispersed suggests that variation in the number of transporters per vesicle is not common. Thus the observation that variation in transporter expression alters the amount of neurotransmitter packaged into vesicles (Wojcik et al., 2004; Daniels et al., 2006) is not likely to be a widespread source of regulation in vivo.

Because we analyzed vesicles of all transmitter classes, we cannot tell whether polydispersity reflects variability across all vesicle classes or arises from the relative abundance of vesicle classes with different, monodispersed numbers of these proteins. Therefore, one exciting possibility is that polydispersity reflects variability between neurotransmitter classes of vesicles, and that this underlies differences in release probability across different classes of synapses.

Although the tetraspan proteins synaptophysin and synaptogyrin are both polydispersed, they differed significantly in their average number per vesicle $(6.8 \pm 0.36$ for synaptogyrin and $13.06 \pm 0.83$ for synaptophysin). Because loss of both synaptophysin and synaptogyrin is required to observe deficits in synaptic functioning (Thiele et al., 2000), it has been assumed that they provide a redundant function. The discrepancy in their average number suggests that this may not be true and that these proteins may perform unique actions in vesicles.

In summary, knowing the number and variance of vesicle proteins provides a basis for vetting models of exocytosis and endocytosis and screening alterations in vesicle composition in animal models of neurological disorders. We anticipate that this approach will find wide use in future studies of synaptic regulation.

\section{References}

Alés E, Tabares L, Poyato JM, Valero V, Lindau M, Alvarez de Toledo G (1999) High calcium concentrations shift the mode of exocytosis to the kiss-and-run mechanism. Nat Cell Biol 1:40-44.

Aravanis AM, Pyle JL, Tsien RW (2003) Single synaptic vesicles fusing transiently and successively without loss of identity. Nature 423:643-647.

Bekkers JM, Richerson GB, Stevens CF (1990) Origin of variability in quantal size in cultured hippocampal neurons and hippocampal slices. Proc Natl Acad Sci U S A 87:5359-5362.

Buckley K, Kelly RB (1985) Identification of a transmembrane glycoprotein specific for secretory vesicles of neural and endocrine cells. J Cell Biol 100:1284-1294.
Burré J, Beckhaus T, Schägger H, Corvey C, Hofmann S, Karas M, Zimmermann H, Volknandt W (2006) Analysis of the synaptic vesicle proteome using three gel-based protein separation techniques. Proteomics 6:6250-6262.

Calakos N, Scheller RH (1994) Vesicle-associated membrane protein and synaptophysin are associated on the synaptic vesicle. J Biol Chem 269:24534-24537.

Ceccarelli B, Hurlbut WP, Mauro A (1973) Turnover of transmitter and synaptic vesicles at the frog neuromuscular junction. J Cell Biol 57:499-524.

Daniels RW, Collins CA, Chen K, Gelfand MV, Featherstone DE, DiAntonio A (2006) A single vesicular glutamate transporter is sufficient to fill a synaptic vesicle. Neuron 49:11-16.

Fernández-Alfonso T, Kwan R, Ryan TA (2006) Synaptic vesicles interchange their membrane proteins with a large surface reservoir during recycling. Neuron 51:179-186.

Fiorini GS, Chiu DT (2005) Disposable microfluidic devices: fabrication, function, and application. Biotechniques 38:429-446.

Gracz LM, Wang WC, Parsons SM (1988) Cholinergic synaptic vesicle heterogeneity: evidence for regulation of acetylcholine transport. Biochemistry 27:5268-5274.

Hayashi M, Raimondi A, O'Toole E, Paradise S, Collesi C, Cremona O, Ferguson SM, De Camilli P (2008) Cell- and stimulus-dependent heterogeneity of synaptic vesicle endocytic recycling mechanisms revealed by studies of dynamin 1-null neurons. Proc Natl Acad Sci U S A 105:2175-2180.

He L, Wu LG (2007) The debate on the kiss-and-run fusion at synapses. Trends Neurosci 30:447-455.

He L, Wu XS, Mohan R, Wu LG (2006) Two modes of fusion pore opening revealed by cell-attached recordings at a synapse. Nature 444:102-105.

Jahn R, Südhof TC (1994) Synaptic vesicles and exocytosis. Annu Rev Neurosci 17:219-246.

Kapitein LC, Kwok BH, Weinger JS, Schmidt CF, Kapoor TM, Peterman EJ (2008) Microtubule cross-linking triggers the directional motility of kinesin-5. J Cell Biol 182:421-428.

Klyachko VA, Jackson MB (2002) Capacitance steps and fusion pores of small and large-dense-core vesicles in nerve terminals. Nature 418:89-92.

Limpert E, Stahel WA, Abbt M (2001) Log-normal distributions across the sciences: keys and clues. Bioscience 51:341-352.

Matveeva EA, Whiteheart SW, Slevin JT (2003) Accumulation of 7S SNARE complexes in hippocampal synaptosomes from chronically kindled rats. J Neurochem 84:621-624.

Matveeva EA, Vanaman TC, Whiteheart SW, Slevin JT (2007) Asymmetric accumulation of hippocampal 7S SNARE complexes occurs regardless of kindling paradigm. Epilepsy Res 73:266-274.

Matveeva EA, Vanaman TC, Whiteheart SW, Slevin JT (2008) Levetiracetam prevents kindling-induced asymmetric accumulation of hippocampal 7S SNARE complexes. Epilepsia 49:1749-1758.

Mirnics K, Middleton FA, Marquez A, Lewis DA, Levitt P (2000) Molecular characterization of schizophrenia viewed by microarray analysis of gene expression in prefrontal cortex. Neuron 28:53-67.

Morciano M, Burré J, Corvey C, Karas M, Zimmermann H, Volknandt W (2005) Immunoisolation of two synaptic vesicle pools from synaptosomes: a proteomics analysis. J Neurochem 95:1732-1745.

Müller M, Goutman JD, Kochubey O, Schneggenburger R (2010) Interaction between facilitation and depression at a large CNS synapse reveals mechanisms of short-term plasticity. J Neurosci 30:2007-2016.

Murthy VN, Stevens CF (1998) Synaptic vesicles retain their identity through the endocytic cycle. Nature 392:497-501.

Mutch SA, Fujimoto BS, Kuyper CL, Kuo JS, Bajjalieh SM, Chiu DT (2007) Deconvolving single-molecule intensity distributions for quantitative microscopy measurements. Biophys J 92:2926-2943.

Parpura V, Doyle RT, Basarsky TA, Henderson E, Haydon PG (1995) Dynamic imaging of purified individual synaptic vesicles. Neuroimage 2:3-7.

Press WH (2001) Numerical recipes in Fortran 77: the art of scientific computing, Ed 2 (with corrections). Cambridge, UK; New York: Cambridge UP.

Richards DA, Bai J, Chapman ER (2005) Two modes of exocytosis at hippocampal synapses revealed by rate of FM1-43 efflux from individual vesicles. J Cell Biol 168:929-939.

Schivell AE, Batchelor RH, Bajjalieh SM (1996) Isoform-specific, calcium- 
regulated interaction of the synaptic vesicle proteins SV2 and synaptotagmin. J Biol Chem 271:27770-27775.

Takamori S, Holt M, Stenius K, Lemke EA, Grønborg M, Riedel D, Urlaub H, Schenck S, Brügger B, Ringler P, Müller SA, Rammner B, Gräter F, Hub JS, De Groot BL, Mieskes G, Moriyama Y, Klingauf J, Grubmüller H, Heuser J, et al. (2006) Molecular anatomy of a trafficking organelle. Cell 127:831-846.

Thiele C, Hannah MJ, Fahrenholz F, Huttner WB (2000) Cholesterol binds to synaptophysin and is required for biogenesis of synaptic vesicles. Nat Cell Biol 2:42-49.

Trommershäuser J, Schneggenburger R, Zippelius A, Neher E (2003) Heterogeneous presynaptic release probabilities: functional relevance for short-term plasticity. Biophys J 84:1563-1579.

Wienisch M, Klingauf J (2006) Vesicular proteins exocytosed and subsequently retrieved by compensatory endocytosis are nonidentical. Nat Neurosci 9:1019-1027.

Willig KI, Rizzoli SO, Westphal V, Jahn R, Hell SW (2006) STED micros- copy reveals that synaptotagmin remains clustered after synaptic vesicle exocytosis. Nature 440:935-939.

Wojcik SM, Rhee JS, Herzog E, Sigler A, Jahn R, Takamori S, Brose N, Rosenmund C (2004) An essential role for vesicular glutamate transporter 1 (VGLUT1) in postnatal development and control of quantal size. Proc Natl Acad Sci U S A 101:7158-7163.

Wu W, Xu J, Wu XS, Wu LG (2005) Activity-dependent acceleration of endocytosis at a central synapse. J Neurosci 25:11676-11683.

Yao J, Nowack A, Kensel-Hammes P, Gardner RG, Bajjalieh SM (2010) Cotrafficking of SV2 and synaptotagmin at the synapse. J Neurosci 30:5569-5578.

Yao PJ (2004) Synaptic frailty and clathrin-mediated synaptic vesicle trafficking in Alzheimer's disease. Trends Neurosci 27:24-29.

Yao PJ, Zhu M, Pyun EI, Brooks AI, Therianos S, Meyers VE, Coleman PD (2003) Defects in expression of genes related to synaptic vesicle trafficking in frontal cortex of Alzheimer's disease. Neurobiol Dis 12:97109. 